\title{
Chinese Herbal Medicine Wenxia Changfu Formula Reverses Cell Adhesion-Mediated Drug Resistance via the Integrin $\beta 1-P I 3 K-A K T$ Pathway in Lung Cancer
}

\author{
YaNan Zhang ${ }^{2,3^{*}}$, ZhiChun $\mathrm{Wu}^{2,3^{*}}$, HuaYun $\mathrm{Yu}^{2,3}$, HuaXin Wang 2,3 , Guowei Liu ${ }^{3}$, ShiJun Wang ${ }^{2,3}{ }^{\bowtie}$, XuMing \\ $\mathrm{Ji}^{1,3}$ \\ 1. College of Basic Medicine,Zhejiang Chinese Medical University, Hangzhou, Zhejiang Province 310053, China. \\ 2. College of Traditional Chinese Medicine, Shandong University of Traditional Chinese Medicine, Jinan, Shangdong Province 250355, China. \\ 3. Shandong Provincial Chinese Medicine Classical Prescription Demonstration Engineering Technology Research Center, Jinan, Shangdong Province 250355, \\ China. \\ *YaNan Zhang and ZhiChun Wu contributed equally to this work.
}

$\triangle$ Corresponding authors: Professor XuMing Ji, PhD; Professor ShiJun Wang, PhD; Tel/Fax: +86-571-86613610; Tel/Fax: +86-531-89628185; E-mail address: jixuming724@163.com; pathology@163.com

() Ivyspring International Publisher. This is an open access article distributed under the terms of the Creative Commons Attribution (CC BY-NC) license (https://creativecommons.org/licenses/by-nc/4.0/). See http://ivyspring.com/terms for full terms and conditions.

Received: 2018.01.26; Accepted: 2018.10.04; Published: 2019.01.01

\begin{abstract}
In the treatment of lung cancer, the multidrug resistance to chemotherapeutic drugs is one of the reasons of low rates for cure and treatment failure, the combination of chemotherapeutic drugs and traditional Chinese medicine can increase the sensitivity of chemotherapy and reduce its adverse effects. Our previous study has proved that Chinese herbal medicine (CHM) Wenxia Changfu Formula (WCF for short) effectively enhances chemotherapeutic efficacy in lung cancer treatment and reverses multidrug resistance in lung cancer cells in vitro. The present study aims to investigate the effect and mechanism of WCF in reversing cell adhesion-mediated drug resistance of lung cancer by using A549 three-dimensional cell culture and nude mouse model of the A549 cell line with Integrin $\beta 1$ overexpression. We show that the combination of WCF with DDP can decrease proliferation of lung cancer cells by inducing cell cycle arrest and apoptosis. Moreover, we find that the combination of WCF with DDP suppresses the expression of certain molecules which regulate cell cycle and apoptosis. Mechanistically, we show that the Integrin $\beta 1, F A K, P I 3 K$, and AKT protein expressions are suppressed by DDP and even more responses are observed when DDP and WCF are combined, showing WCF treatment enhances the effect of commonly used anticancer drugs. In line with the above findings, our results confirm that WCF reverses cell adhesion-mediated drug resistance of lung cancer via inactivating Integrin $\beta 1 / \mathrm{PI} K / \mathrm{AKT}$ and apoptosis induction.
\end{abstract}

Key words: lung cancer, traditional Chinese medicine, combination therapy, integrin $\beta 1$

\section{Introduction}

Lung cancer is the most common cancer in terms of incidence and death rate in China [1]. In 2015 alone, 733,300 new cases and 610,200 deaths from lung cancer were estimated [2]. Currently, chemotherapy still remains the main treatment for lung cancer patients [3]. However, resistance to chemotherapeutic drugs can result in low rates for cure and treatment failure [4]. Therefore, preventing or inhibiting chemoresistance becomes a rationalized approach for lowering high mortality among lung cancer patients.

The combination of traditional Chinese medicine and chemotherapy drugs is a therapy method with
Chinese characteristics. The traditional Chinese medicine can increase the sensitivity of chemotherapy and reduce its adverse effects. In our earlier studies, we have found that WCF improves the sensitivity of tumor cells to CDDP and reverses the drug resistance [5]. However, the detailed molecular mechanism underlining this has not been fully elucidated.

Now, there are ample evidences that tumor microenvironment provides sanctuary for lung cancer cells [6]. The adhesion of tumor cells to microenvironmental components directly causes tumor cells to become less sensitive to chemotherapeutic drugs, 
which is called cell adhesion mediated drug resistance (CAM-DR) [7]. Therefore, intervention of lung cancer microenvironment mediated CAM-DR may improve the clinical efficacy of chemotherapeutic agents.

During CAM-DR, the activation of integrins promotes tumor cells adhesion to extracellular matrix (ECM) proteins [8]. Increasing evidence has suggested that the Integrin $\beta 1$ has a significant role in mediating resistance to chemotherapies by enhancing cell survival in myeloma, glioblastoma, ovarian cancer and lung cancer $[9,10,11]$. Integrin $\beta 1$ recognizes and binds specific ligands (such as fibronectin, vitornectin, collagen and laminin) resulting in clustering of integrins and recruitment and activation of signaling/ adaptor molecules such as Phosphatidylinositol 3-kinase (PI3K)/ protein kinase B (AKT) [12]. PI3K/ AKT signaling, which is a prototypic survival pathway, plays a stimulatory role in proliferation, survival, and drug-resistance in many cancer types $[13,14]$. Therefore, it is expected that targeting integrin $\beta 1$ expression and downstream PI3K/AKT signaling molecules through Chinese herbal medicine should improve lung cancer cell sensitivity to platinum based chemotherapy.

Accordingly, in the present study, we examined the effect of WCF on reversing the cell adhesionmediated drug resistance and analyzed the potential mechanism by using A549 three-dimensional cell culture and nude mouse model of the A549 cell line with Integrin $\beta 1$ overexpression. Results clearly showed that WCF sensitized the inhibition effect of DDP on lung cancer cells growth via inactivating Integrin $\beta 1 / \mathrm{PI} 3 \mathrm{~K} / \mathrm{AKT}$ and apoptosis induction.

\section{Materials and Methods}

\section{Chinese Herbal Medicine}

The Chinese Herbal Medicine WCF prescribed by Dr. Feng Li in Shandong University of Traditional Chinese Medicine was provided by Affiliated Hospital of Shandong University of Traditional Chinese Medicine. Radix Aconiti Praeparatae(12 g) and Panax ginseng $(9 \mathrm{~g})$ were macerated for $1 \mathrm{~h}$, decocted for $2 \mathrm{~h}$, and added Angelica sinensis $(6 \mathrm{~g})$, decocted for $1 / 2 \mathrm{~h}$, then added Radix et Rhizoma Rhei(12 g), decocted for $1 / 4 \mathrm{~h}$ for the first and the second time. The filtrates were mixed and condensed to $2 \mathrm{~g}$ crude drug/ $\mathrm{mL}$, stored at $4{ }^{\circ} \mathrm{C}$.

\section{Drug Contained Serum Preparation}

The rats were randomly divided into medicated serum group and normal serum group. For the medicated serum group, rats were given WCF $(0.35$ $\mathrm{g} / \mathrm{kg}$ ) by gavage, while rats of the normal serum group were given the same volume of saline 2 times per day for 3 days. Rats were fasted $12 \mathrm{~h}$ after the last administration and then given WCF 1 day's dosage. Blood was taken after $1 \mathrm{~h}$ and inactivated at $63.5^{\circ} \mathrm{C}$ and made into freeze-dried powder stored at $-70^{\circ} \mathrm{C}$.

\section{Reagents and Cell Culture}

A549 cell line was purchased from the Cell Bank of Type Culture Collection of Chinese Academy of Sciences (Shanghai, China). A549 cells were maintained in RPMI 1640 (Pharmacia, Sweden) supplemented with $10 \%$ calf serum ( $\mathrm{Si}$ ji qing bioengineering Material Co., Ltd., Hangzhou, China) and antibiotics (penicillin, $100 \mathrm{U} \mathrm{mL}^{-1}$; streptomycin $100 \mu \mathrm{g} \mathrm{mL}-1$ ) at $37^{\circ} \mathrm{C}$ in a humidified atmosphere of $5 \% \mathrm{CO}_{2}$. The cell concentration was adjusted to $5.5 \times 10^{6} / \mathrm{mL}$, and then added into 24 well plate which has laid the agarose gel for three-dimensional cell culture.

\section{Cell viability assay}

Cell viability was measured using the CellTiter 96 AQueous One Solution Cell Proliferation Assay. Briefly, the A549 cells from three-dimensional cell culture system were seeded into a 96-well plate at $5 \times 10^{3}$ cells/well, and incubated at $37^{\circ} \mathrm{C}$ with $5 \% \mathrm{CO}_{2}$ for $48 \mathrm{~h}$ before treated. A549 cells were randomly divided into 4 groups: control group, DDP group (addition of normal serum and $10 \mu \mathrm{g} / \mathrm{ml}$ DDP), combination of WCF medicated serum and DDP group (addition of WCF medicated serum, and $10 \mu \mathrm{g} / \mathrm{ml} \mathrm{DDP}$ ), and LY294002 group (addition of LY294002, $10 \mathrm{umol} / \mathrm{L})$.

After respectively treatment for $24 \mathrm{~h}$, cell viability was measured using the CellTiter 96 AQueous One Solution Cell Proliferation Assay according to the manufacturer's instructions. Absorbance at $490 \mathrm{~nm}$ was determined using ELISA reader (SpectraMax M3, Molecular Devices, USA). Cell viability (\% of control) was calculated as (absorbance of test sample/ absorbance of control) $\times 100 \%$.

\section{Cell cycle analysis}

Cells $\left(4 \times 10^{5}\right.$ cells $\left./ \mathrm{ml}\right)$ in the logarithmic growth phase were seeded into six well plates. The A549 cells from three-dimensional cell culture system were incubated for $24 \mathrm{~h}$ in serum free medium (Gibco; Thermo Fisher Scientifc, Inc.) to arrest the cell cycle in the G0 phase. The cells were then incubated in DMEM (Gibco; Thermo Fisher Scientifc, Inc.) supplemented with $10 \%$ fetal bovine serum (Gibco; Thermo Fisher Scientifc, Inc.). After $48 \mathrm{~h}$ incubation, the A549 cells were treated for $24 \mathrm{~h}$ with DDP (addition of normal serum and $10 \mu \mathrm{g} / \mathrm{ml} \mathrm{DDP}$ ), combination of WCF medicated serum and DDP (addition of WCF medicated serum and 10 $\mathrm{gg} / \mathrm{ml}$ DDP), and LY294002 (addition of LY294002, $10 \mathrm{umol} / \mathrm{L}$ ) respectively. Cells were harvested by trypsinization, fixed in $70 \%$ 
ice-cold ethanol for $2 \mathrm{~h}$ and stained with propidium iodide (Beijing Biosea Biotechnology Co. Ltd., Beijing, China). Cell cycle analysis was performed using a FC500 flow cytometer (Beckman Coulter, Inc., Brea, CA, USA).

\section{Apoptosis analysis assays}

Apoptosis was analyzed using the Annexin V-FITC Apoptosis Detection Kit according to the manufacturer's instructions. Cells from threedimensional cell culture system were seeded into 6-well plates. Cells with DDP (addition of normal serum and $10 \mu \mathrm{g} / \mathrm{ml} \mathrm{DDP}$ ), combination of WCF medicated serum and DDP (addition of WCF

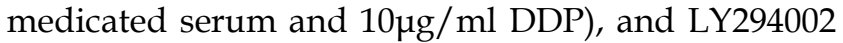
(addition of LY294002, $10 \mathrm{umol} / \mathrm{L}$ ) respectively treatment for $24 \mathrm{~h}$, which were harvested, washed with ice-cold PBS for twice, and resuspended in 1000 $\mu \mathrm{L}$ binding buffer containing $10 \mu \mathrm{L}$ Annexin V-FITC staining solution. The cells were incubated in the dark for $15 \mathrm{~min}$, and then added $5 \mu \mathrm{L}$ PI staining solution. Then the cells were gently mixed and incubated for 15 min in the dark at room temperature, stained cells were immediately analyzed. Apoptosis was detected using a flow cytometer. The data were analyzed with the CFlow Plus software (Becton Dickinson). All the samples were assayed in triplicate.

\section{Immunofluorescence}

Cells from three-dimensional cell culture system were seeded into 6-well plates. Cells with DDP (addition of normal serum and $10 \mu \mathrm{g} / \mathrm{ml}$ DDP), combination of WCF medicated serum and DDP (addition of WCF medicated serum and $10 \mu \mathrm{g} / \mathrm{ml}$ DDP), and LY294002 (addition of LY294002, 10 umol/L) respectively treatment for $24 \mathrm{~h}$, which were harvested, washed with PBS and fixed in $4 \%$ paraformaldehyde for $10 \mathrm{~min}$. Permeabilisation and blocking were done with $2 \%$ FBS in $0.3 \%$ Triton X-100 (Calbiochem) in PBS followed by probing with the primary antibody to P21(1:1000; lot number: Ab109520, Abcam, USA), and incubation overnight at $4^{\circ} \mathrm{C}$. The cells were washed with $0.05 \%$ Tween 20 in PBS and added secondary antibodies labeled with labeled by fluorescence for $1 \mathrm{~h}$ in a dark moist chamber. The slide was then mounted on gold antifade reagent with DAPI and examined under a fluorescent microscope (Olympus BX51). The images were scanned by laser scanning confocal microscope, and each sample randomly selected 30 cells to measure the fluorescence intensity of the cells. Integrated option density were analyzed and quantified by using scientific imaging software (Image J).

\section{Western blotting analysis}

For Western blotting, the cells were harvested, washed and lysed with $1 \times$ RAPI buffer. Protein quantification was determined by the Bradford protein assay, and samples were equally loaded on $10 \%$ polyacrylamide gels electrophoresed at $200 \mathrm{~V}$, electrotransferred to polyvinylidene fluoride membranes, and probed with antibodies to Integrin B1(1:1000; lot number: Ab179471, Abcam, USA), FAK (1:1000; lot number: Ab40794, Abcam, USA), PI3K (1:1000; lot number: Ab40755, Abcam, USA), AKT (1:1000; lot number: Ab25893, Abcam, USA), GSK-3 $\beta$ (1:1000; lot number: Ab93926, Abcam, USA ), Cyclin D1 (1:1000; lot number: Ab134175, Abcam, USA), $\operatorname{BAD}$ (1:1000; lot number: Ab32445, Abcam, USA). The images were scanned, and blots were quantified by densitometry using scientific imaging software (Image J) and normalized to loading controls.

\section{qRT-PCR analysis}

The total RNA was isolated using Trizol reagent and reverse-transcribed into cDNA using the TaKaRa RT reagent kit. The PCR reactions were quantified by ABI 7500 fast qRT-PCR System. The PCR conditions were as follows: $30 \mathrm{~s}$ at $95^{\circ} \mathrm{C}$, followed by 40 cycles of $5 \mathrm{~s}$ at $95^{\circ} \mathrm{C}$, and $34 \mathrm{~s}$ at $60^{\circ} \mathrm{C}$. Each sample was tested in triplicate. Threshold values were determined for each sample/primer pair, and the average and standard errors were calculated. Cycle threshold $(\mathrm{Ct})$ values were obtained graphically for the target genes and $\beta$-actin. $\Delta \mathrm{Ct}=\mathrm{Ct}$ (target genes $)-\mathrm{Ct}(\beta$-actin $) . \Delta \Delta \mathrm{Ct}=$ $\Delta \mathrm{Ct}$ (treated samples) $-\Delta \mathrm{Ct}$ (control samples). The relative fold change in gene expression was calculated as $2^{-\triangle \Delta C T}$.

A quantitative real-time PCR (qRT-PCR) assay was developed for the detection and quantification of Integrin $\beta 1$, FAK, PI3K, AKT, GSK-3 $\beta$, Cyclin D1, BAD transcripts using $\beta$-actin as an endogenous control. The sequences of the primers are summarized in Table 1.

Table 1. Sequences for Real-time RT-PCR Primers

\begin{tabular}{lll}
\hline Gene & Forward (5/-3/) & Reverse (5/-3/) \\
\hline $\begin{array}{l}\text { Integrin } \\
\beta 1\end{array}$ & GTGTTTCCACGGATGCT & GTGTGCCCACTGCTGACT \\
FAK & AATATGAACTCGCCCACCCAC & CGCCTAATTTCCAAACAACCC \\
PI3K & ATTTCCAGTGGGTGAGGCAG & CTCATGGTAGCCGGTGACTC \\
AKT & TACGGTGCGGAGATTGTGTC & ACAGCCCGAAGTCCGTTATC \\
GSK-3 $\beta$ & CGAACTCCACCAGAGGCAAT & AAGAGTGCAGGTGTGTCTCG \\
Cyclin D1 & AAGGGCACAAGTCCTGGATG & TGCTGGAAACATGCCGGTTA \\
BAD & CGGGCAGCCACCAACAGTCAT & ACGCGAGCGTCCTCGAAAAGG \\
$\beta$-actin & ACTCTGTGTGGATTGGTGGC & AGAAAGGGTGTAAAACGCAGC \\
\hline
\end{tabular}

\section{Animal Studies}

To further explore the effects and mechanisms of DDP and the combination of DDP and WCF on tumor 
growth in vivo, we established a nude mouse model of the A549 cell line with Integrin $\beta 1$ overexpression (A549/ D6). A total of 30 STOCK-Foxn1nu/ NJu female nude mice (four-week-old) were purchased from Model Animal Research Center of Nanjing University and were maintained at the Animal experimental center of Shandong University of Traditional Chinese Medicine in a specific pathogen-free environment with food and water provided. All experiments were conducted with the approval of the Institutional Animal Care and Use Committee and were in compliance with the National Institutes of Health Guidelines for Use and Care of Laboratory Animals.

A549 cells with Integrin $\beta 1$ overexpression (A549-D6, obtained from Shanghai Genechem Co., Ltd, Shanghai, China) $\left(1.5 \times 10^{6}\right.$ cells $)$ in $100 \mu \mathrm{L}$ PBS were injected subcutaneously into the right armpit of female nude mice. Mice of control group received saline injections on the same day of tumor inoculation.

Mice were randomly divided into the control group, DDP group, combination of WCF and DDP group. On the same day of tumor inoculation, mice of DDP group were given DDP $(8 \mathrm{mg} / \mathrm{ml})$ by peritoneal injection twice a week; The mice of combination of WCF and DDP group were given DDP $(8 \mathrm{mg} / \mathrm{ml})$ by peritoneal injection twice a week, at the same time given WCF concentrated liquid $\left(40 \mathrm{mg}^{\mathrm{kg}}{ }^{-1}\right)$ by gavage; The control group were filled with equal doses of normal saline. Each group was treated for four weeks. The calculated formula between human and mouse according to the body surface area is mouse dose $(\mathrm{g} / \mathrm{kg})=$ human dose $(\mathrm{g} / \mathrm{kg}) \times 3 / 37$.

All mice were euthanized on day 30 , and tumors were removed. Tumor volume measurements were calculated using the formula for an oblong sphere: volume $=1 / 2 \times$ width $^{2} \times$ length. Xenografts of nude mice were prepared as paraffin-embedded sections. Paraffin sections were blocked by a blocking buffer for 1 hour at room temperature and stained with a specific primary antibody for 24 hours. The primary antibody was washed using PBS. The sections then were stained with a specific secondary antibody for 24 hours at room temperature and washed with PBS. The primary antibody used here is listed as follows: P21 (1 : 1000; lot number: Ab109520, Abcam, USA). The protein and mRNA expressions of Integrin $\beta 1, F A K$, PI3K, AKT, GSK-3 $\beta$, CyclinD1, BAD in tumor tissue was detected by Western blot and qRT-PCR.

\section{Statistical analysis}

All data were expressed as means \pm SD. Statistical significance was determined by one-way
ANOVA followed by Bonferroni's multiple comparison test (SPSS 22.0). Differences were considered statistically significant when $\mathrm{P}<0.05$.

\section{Results}

\section{WCF decreased the viability of A549 cells}

We first detected the effect of WCF on cell growth in A549 cells by MTT assay. As shown in Fig. 1A, compared with the control cells, DDP and LY294 002 decreased the cell viability, whereas combination of WCF medicated serum and DDP group showed a further inhibition of cell growth compared with DDP and LY294002 only. The results suggested that the combination of WCF medicated serum and DDP enhanced the growth inhibition in lung cancer cells.

\section{WCF induced cell cycle arrest in A549 cells}

To further examine the effect of WCF on cell proliferation, we performed the flow cytometry of cell cycle analysis. The results showed that, DDP and LY294002 induced G1 phase arrest, and decreased S phase significantly, but no significant changes were found in the G2 phase in A549 cells. Further, the changes of cell cycle distribution of A549 cells treated with combination of WCF medicated serum and DDP were evaluated by flow cytometry (Figs. 1B and 1C). The results revealed that G1 phase arrest was observed in the combination of WCF medicated serum and DDP group.

\section{WCF decreased the protein expression of p2 1}

We examined the expression of P21 in A549 cells by immunofluorescence. It indicated that the level of P21 protein expression was high in A549 cells, whereas DDP and LY294002 decreased P21 expression. Meanwhile, combination of WCF medicated serum and DDP group further decreased P21 expression. Compared with DDP and LY294002 only, the level of P21 protein expression decreased, but there was no statistically significant difference (Figs. $2 \mathrm{~A}$ and $2 \mathrm{~B})$.

\section{WCF promoted the apoptosis of A549 cells}

To further study the effects of WCF, annexin-V/ PI double staining was performed to evaluate the occurrence of apoptosis. Our results showed that, compared with control group, the apoptosis rate of A549 cells in DDP group, combination of WCF medicated serum and DDP group, and LY294002 group increased. Compared with DDP group, combination of WCF medicated serum and DDP induced signifcantly apoptosis in A549 cells, and the effect was better than LY294002 (Figs. 3A and 3B). 

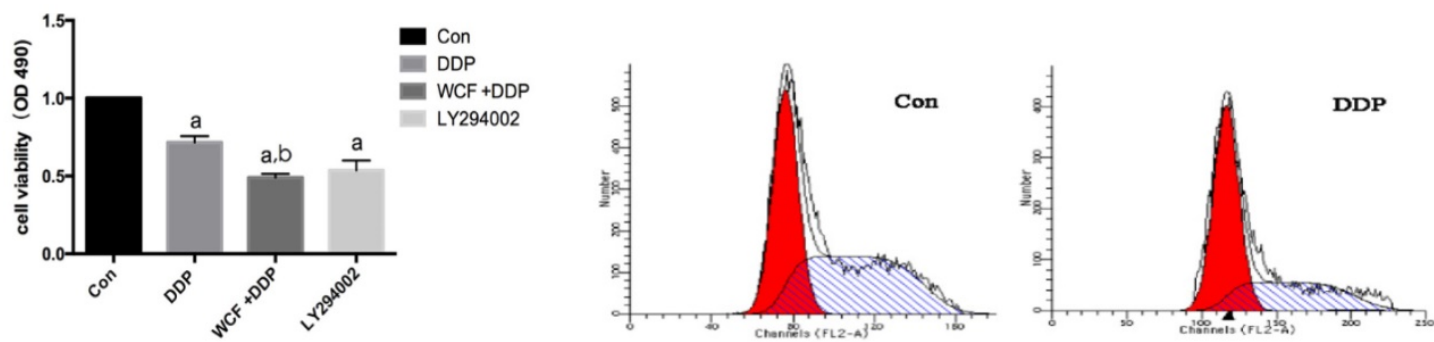

(A)
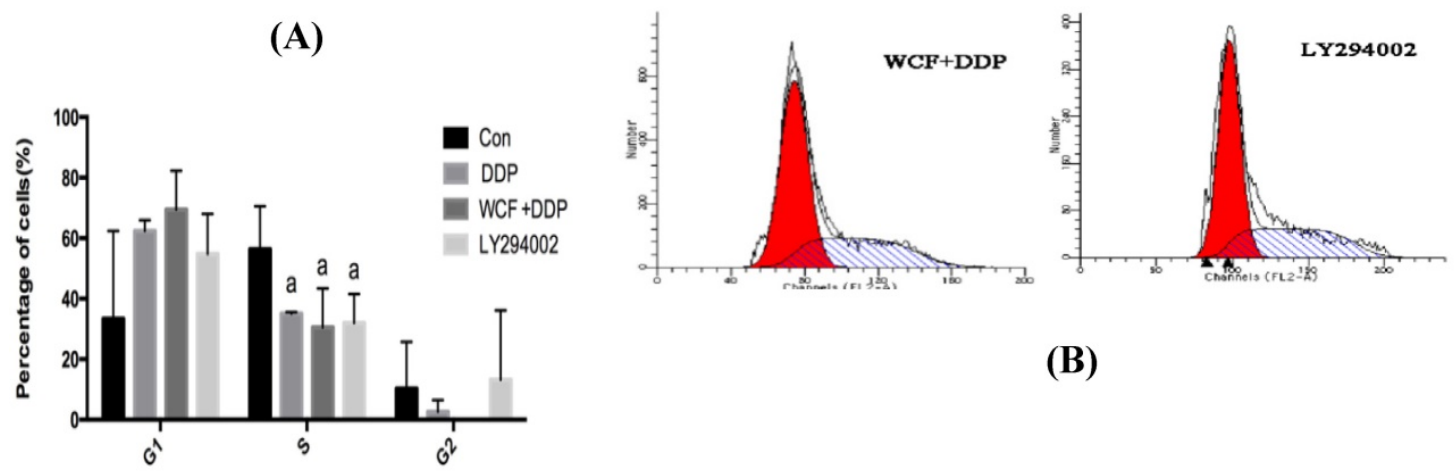

(B)

(C)

Figure 1. WCF inhibited growth and induced cell cycle arrest in lung cancer cells. (A) A549 cells were treated with DDP, combination of WCF medicated serum and DDP for up to $24 \mathrm{~h}$ to examine the cell viability. (B) A549 cells were treated with DDP, combination of WCF medicated serum and DDP for up to $24 \mathrm{~h}$. Cell cycle was measured by flow cytometry. (C) The percentages of the cell population in each phase (G1, S, and G2) were assessed by Multicycle AV DNA Analysis software. Values are given as the mean \pm SD from 3 independent experiments and expressed as a percentage of total cells. aP<0.05, compared with control group. bP<0.05, compared with the DDP Group.

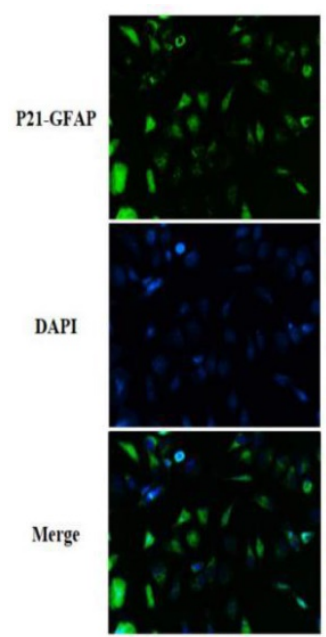

Con

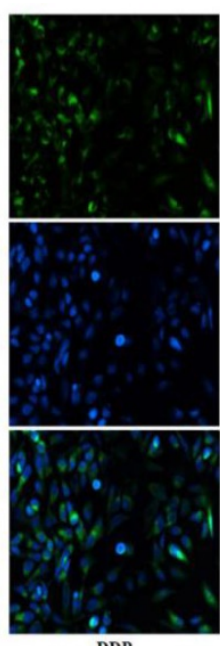

DDP

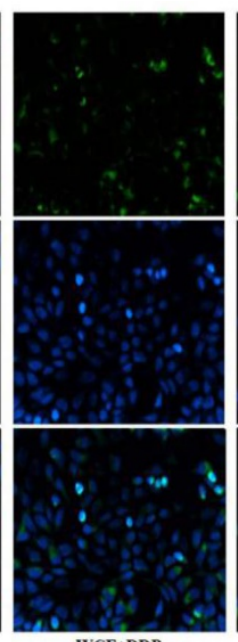

WCF+DDP

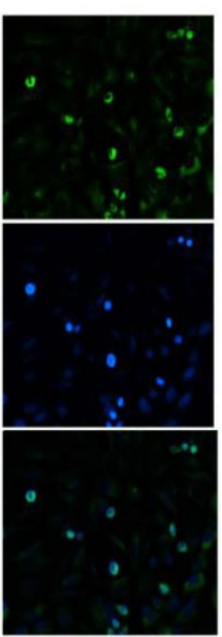

LY294002

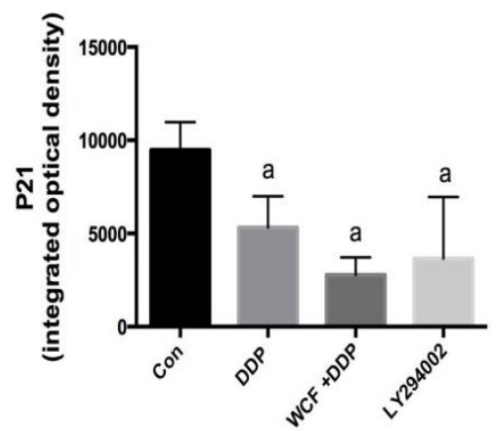

(B)

(A)

Figure 2. Immunofluorescence analysis to detect levels of P21 protein expression of A549 cells in control group, DDP group, combination of WCF medicated serum and DDP group and LY294002 group. aP $<0.05$, compared with control group.

\section{WCF induces cell cycle arrest and apoptotic molecules primarily by Integrin $\beta$ I/PI3K/AKT signaling pathways}

To further explore the effects and molecular mechanisms of inhibition of the tumor growth of the WCF, we employed Western blotting and qRT-PCR to detect the cell cycle, apoptosis, and Integrin $\beta 1 /$ PI3K/AKT pathway related proteins and genes.
As a key regulator of cell cycle, Cyclin D1 was observed by western blotting and qRT-PCR. The results showed that there was no significant effect on Cyclin D1 in response to DDP compared to untreated control cells. Interestingly, the downregulation of Cyclin D1 were observed by treatment of WCF and DDP in A549 cells. Meanwhile, LY294002 significantly decreased the expression of Cyclin D1 in A549 cells. 
Then, apoptosis was analyzed with respect to expression of pro-apoptotic protein, compared with control group, the expression levels of Bad were found to be highly upregulated in DDP group, combination of WCF medicated serum and DDP group and LY294002 group. Compared with the DDP group, combination of WCF and DDP significantly increased the expression of Bad, and the expression of Bad was higher than that of LY294002 group (Figure 4). These results suggested that apoptosis was promoted by WCF treatment.
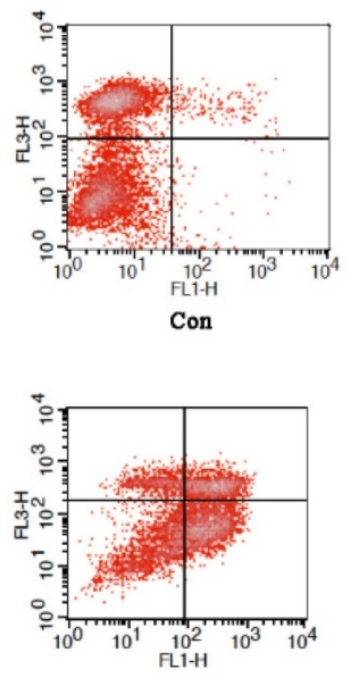

WCF+DDP
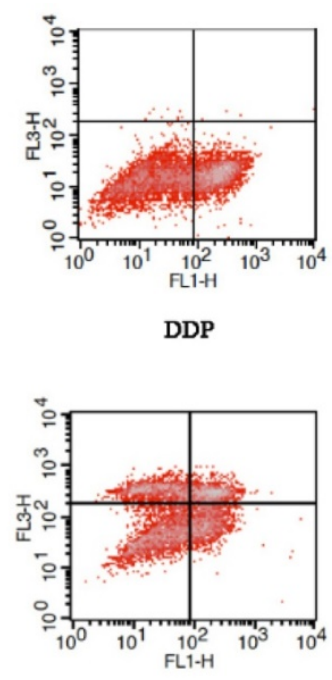

LY294002

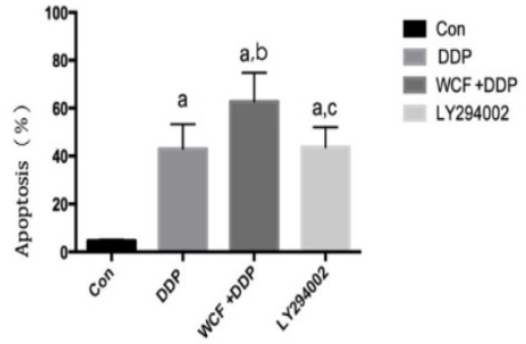

(B)

(A)

Figure 3. Flow cytometric analysis to detect the rates of apoptosis in control group, DDP group, combination of WCF medicated serum and DDP group and LY294002 group. (A) Cell apoptosis stained with Annexin V-FITC/PI was measured by flow cytometry. (B) The histogram depicted the mean apoptotic rate. Values are expressed as Mean \pm SD of eight mice. aP<0.05, compared with control group; ${ }^{\circ}<<0.05$, compared with the DDP Group; $C P<0.05$, compared with combination of WCF medicated serum and DDP group.
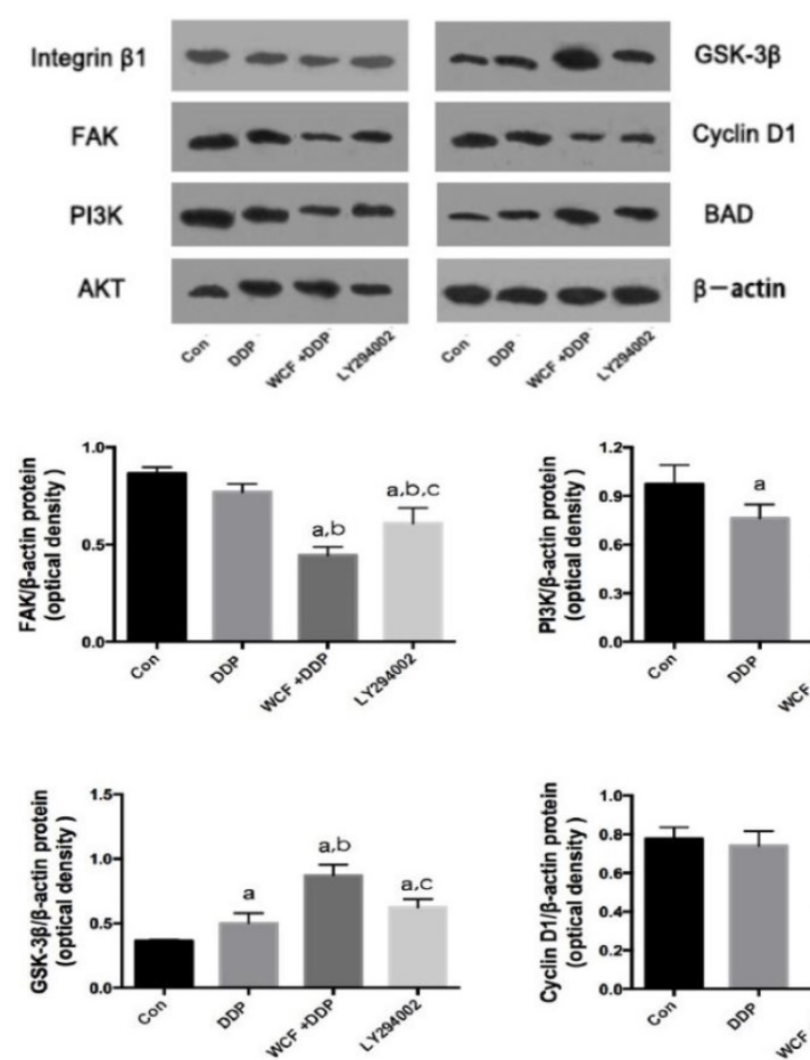
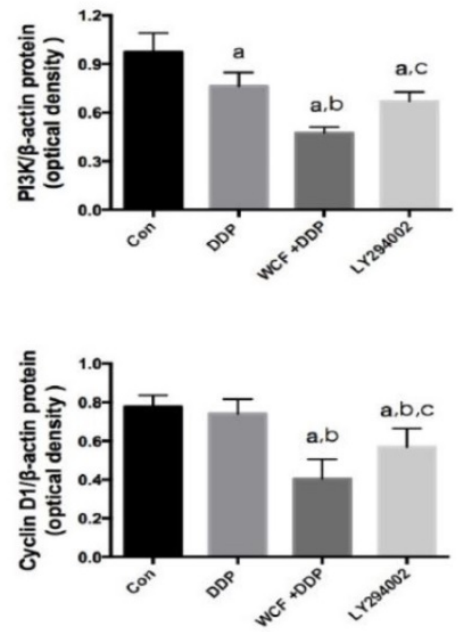
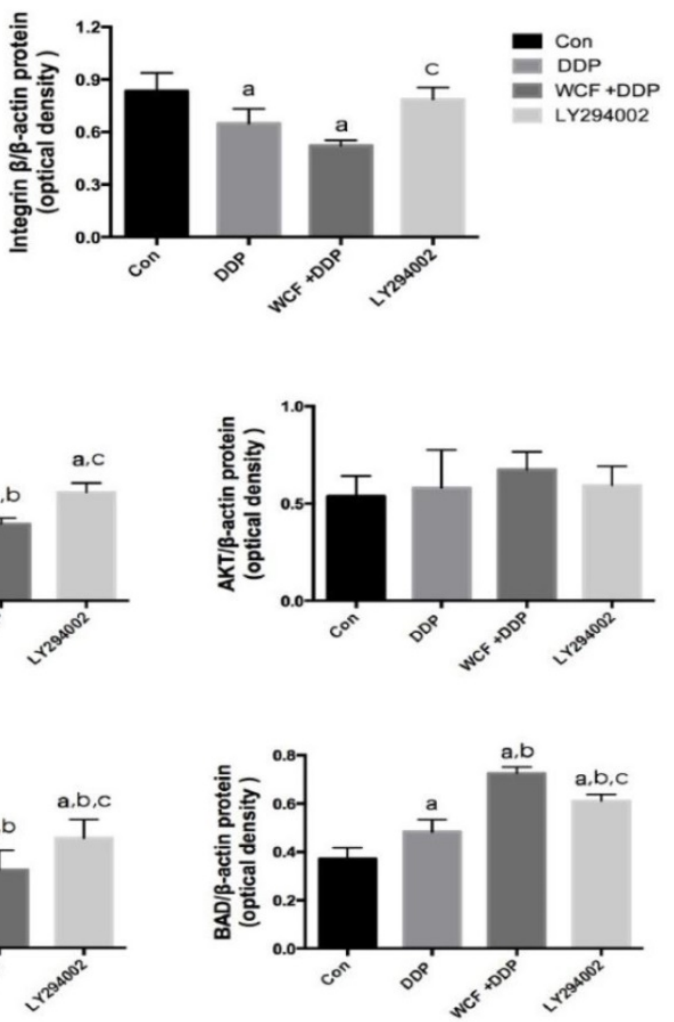

Figure 4. Western blot analysis for expressions of Integrin $\beta 1$, FAK, PI3K, AKT, GSK-3ß, Cyclin DI, BAD in control group, DDP group, combination of WCF medicated serum and DDP group and LY294002 group. The graph represents relative densitometric intensity of each band normalized to $\beta$-actin. Values are expressed as Mean \pm SD of eight mice. aP $<0.05$, compared with control group; P $<0.05$, compared with the DDP Group; $C P<0.05$, compared with combination of WCF medicated serum and DDP group. 

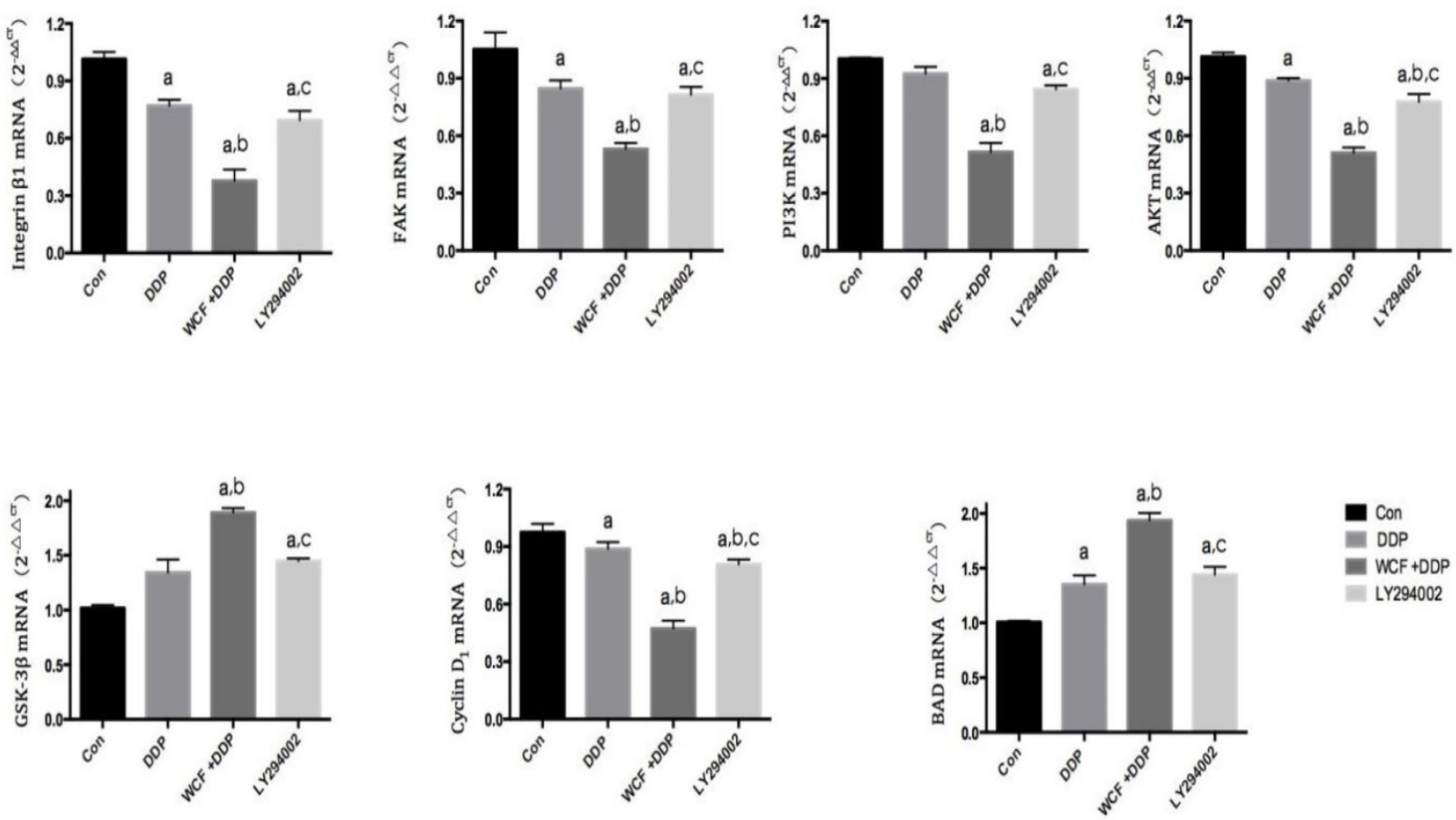

LY294002

Figure 5. qRT-PCR analysis for expressions of Integrin $\beta 1, F A K, P I 3 K, A K T$, GSK-3 $\beta$, Cyclin D1, BAD in control group, DDP group, combination of WCF medicated serum and DDP group and LY294002 group. Values are expressed as Mean \pm SD of eight mice. aP $<0.05$, compared with control group; bP<0.05, compared with the DDP Group; CP $<0.05$, compared with combination of WCF medicated serum and DDP group.

We found that the expressions of Integrin $\beta 1$ and PI3K decreased in DDP group, combination of WCF medicated serum and DDP group, and LY294002 group. Intriguingly, combination of WCF medicated serum and DDP further reduced the expression of Integrin $\beta 1$ and PI3K. But there was not effect on the expressions of AKT. Meanwhile, the levels of GSK-3 $\beta$ increased in DDP group, combination of WCF medicated serum and DDP group, and LY294002 group. Intriguingly, combination of WCF medicated serum and DDP further increased the protein expression of GSK-3 $\beta$ (Figure 4, 5). These results suggested that combination of WCF medicated serum and DDP had effects on cell cycle, apoptosis and Integrin $\beta 1 / \mathrm{PI} 3 \mathrm{~K} / \mathrm{AKT}$ pathway.

\section{Combination treatment of WCF and DDP in a xenograft nude mice model}

We then tested the therapeutic efficacy of DDP and the combination of WCF with DDP on the growth of the A549 cells with integrin $\beta 1$ overexpression (A549/D6) in xenograft nude mice model. We found that, compared to the control group, the DDP treatment group and the combination of WCF and DDP treatment group, showed a significant growth-inhibitory effect. While compared to the DDP treatment group, the combination of WCF and DDP treatment group showed a significant growthinhibitory effect, and the effect was better than the DDP treatment group. The simultaneously determined body weight of the nude mice and tumor volume curves are shown in Fig 6.

To further verify the signal transduction pathway mediating the WCF-induced apoptosis and anti-proliferation effects in vivo, xenografts of nude mice were prepared as paraffin-embedded sections. The immunohistochemical analysis revealed that compared with control group, the expression level of the P21 protein significantly downregulated in DDP group, combination of WCF and DDP treatment group (Figure 7).

We then detected the expression of tissue and found that DDP treatment group decreased the expression of Integrin $\beta 1$, FAk, PI3K, Akt, Cyclin D1. While compared to the DDP treatment group, the combination of WCF and DDP treatment group significantly decreased the expression of Integrin $\beta 1$, FAk, PI3K, Akt, Cyclin D1. Simultaneously, we noticed that expressions of GSK-3 $\beta$ and BAD were increased in the DDP treatment group and the combination of WCF and DDP treatment group as compared to that of the control groups. Compared to the DDP treatment group, the expression of GSK-3 $\beta$ and BAD in combination of WCF and DDP treatment group increased remarkably. Our data demonstrated that WCF induce the apoptosis of A549 cells through integrin $\beta 1$, followed by reducing the expression of PI3K, Akt (Figure 8, 9). 

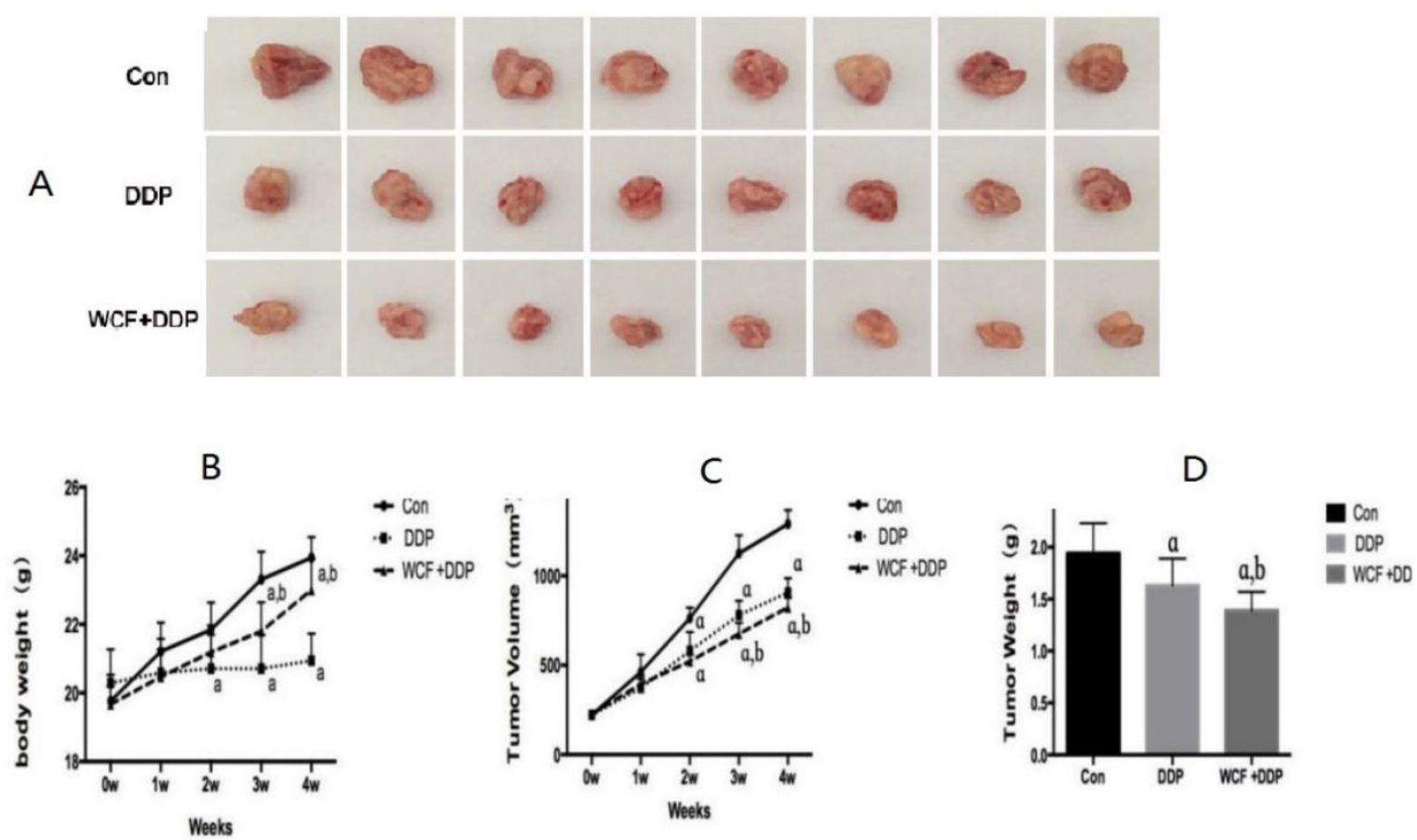

Figure 6. The combination of WCF and DDP suppressed the solid tumor growth of xenograft nude mice model. (A) The tumors were excised from animals after treatment. (B) Changes in body weight in control group, DDP group, combination of WCF and DDP group were measured on weeks $1,2,3,4$. (C) Changes in tumor volume in control group, DDP group, combination of WCF and DDP group were measured on weeks 1, 2, 3, 4. (D) Tumor weights in control group, DDP group, combination of WCF and DDP group were measured on day 29-post tumor cells inoculation. Values are expressed as Mean \pm SD of eight nude mice. aP $<0.05$, compared with control group; bP $<0.05$, compared with the DDP group.
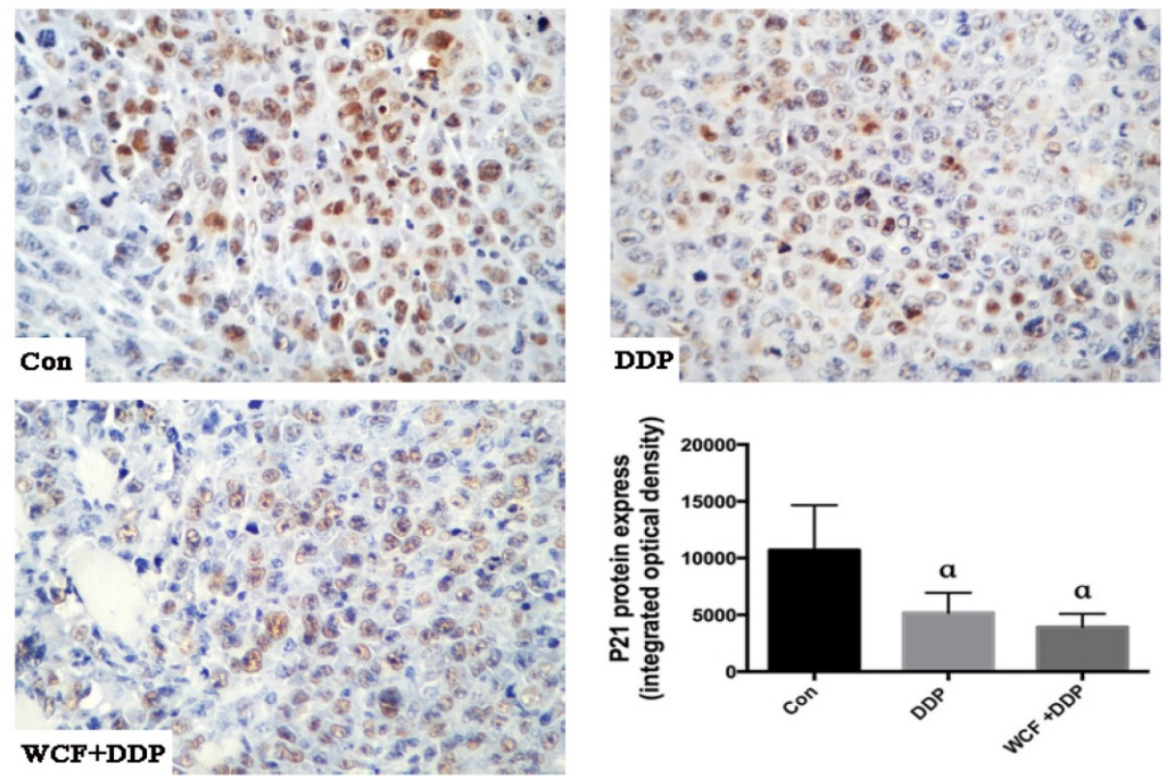

Figure 7. Immunohistochemistry analysis to detect expression levels of P21 protein of lung cancer xenograft in nude mice in control group, DDP group, combination of WCF and DDP group. Values are expressed as Mean \pm SD of eight nude mice. aP $<0.05$, compared with control group.

\section{Discussion}

Despite the advent of several novel therapeutics, the 5-year survival of lung cancer patients still remains low, which is mainly due to the ultimate acquisition of drug resistance [15]. Recent studies have revealed that the tumor microenvironment which provides lung cancer cells a network of proliferative and survival factors is one of the most important reasons for drug resistance and relapse $[16,17]$. However, in the current clinical practice, it is still unable to find a ideal drug to reverse CAM-DR.

Traditional Chinese medicine has been paid more and more attention because of the high efficiency and multiple targets [18]. WCF is composed of Radix Aconiti Praeparatae, Radix et Rhizoma Rhei, 


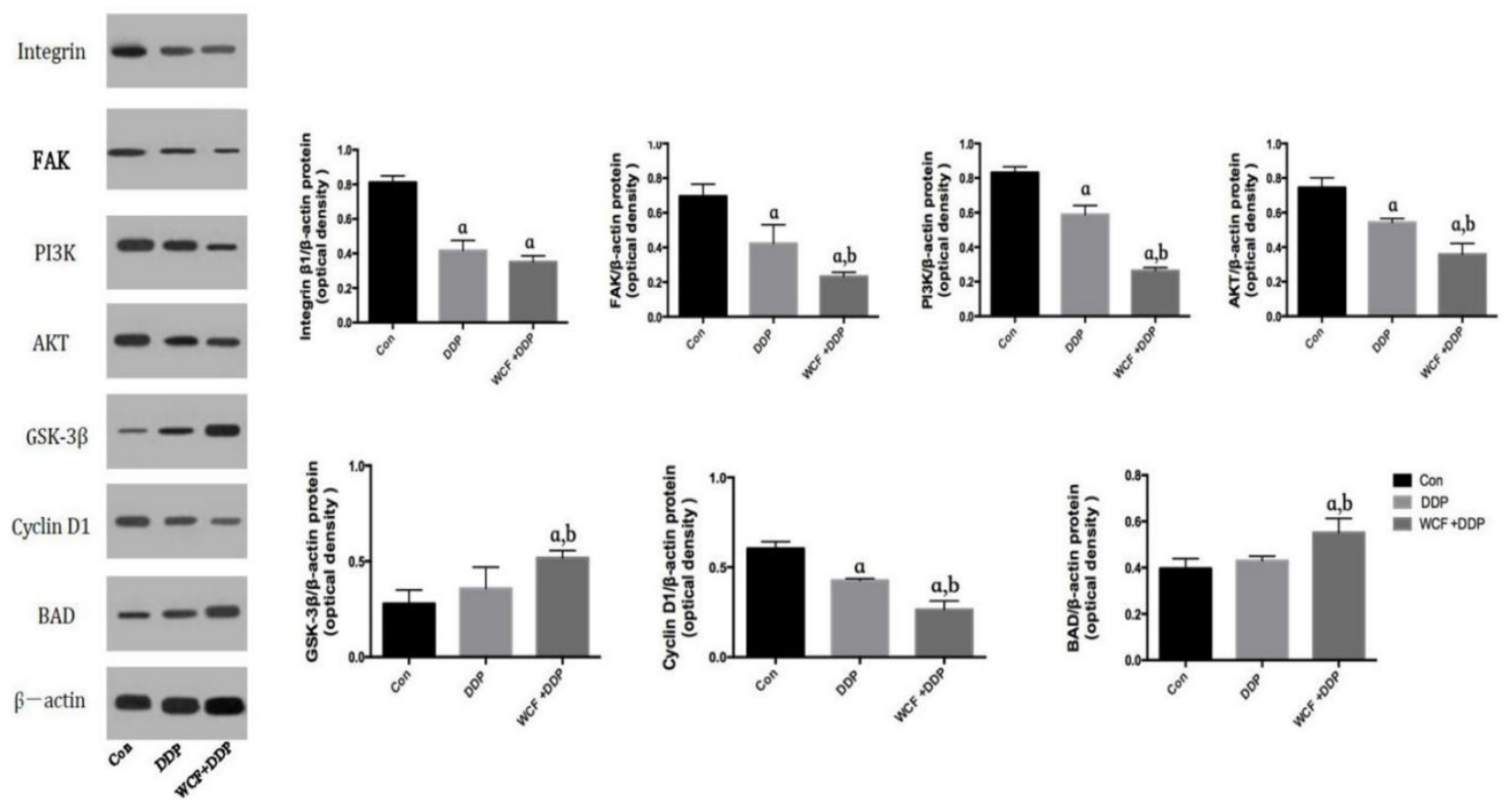

Figure 8. Western blot analysis for expressions of Integrin $\beta 1$, FAK, PI3K, AKT, GSK-3ß, CyclinD1, BAD in nude mice model. The graph represents relative densitometric intensity of each band normalized to $\beta$-actin. Values are expressed as Mean \pm SD of eight mice. aP<0.05, compared with control group; ${ }^{\circ}<<0.05$, compared with the DDP group.
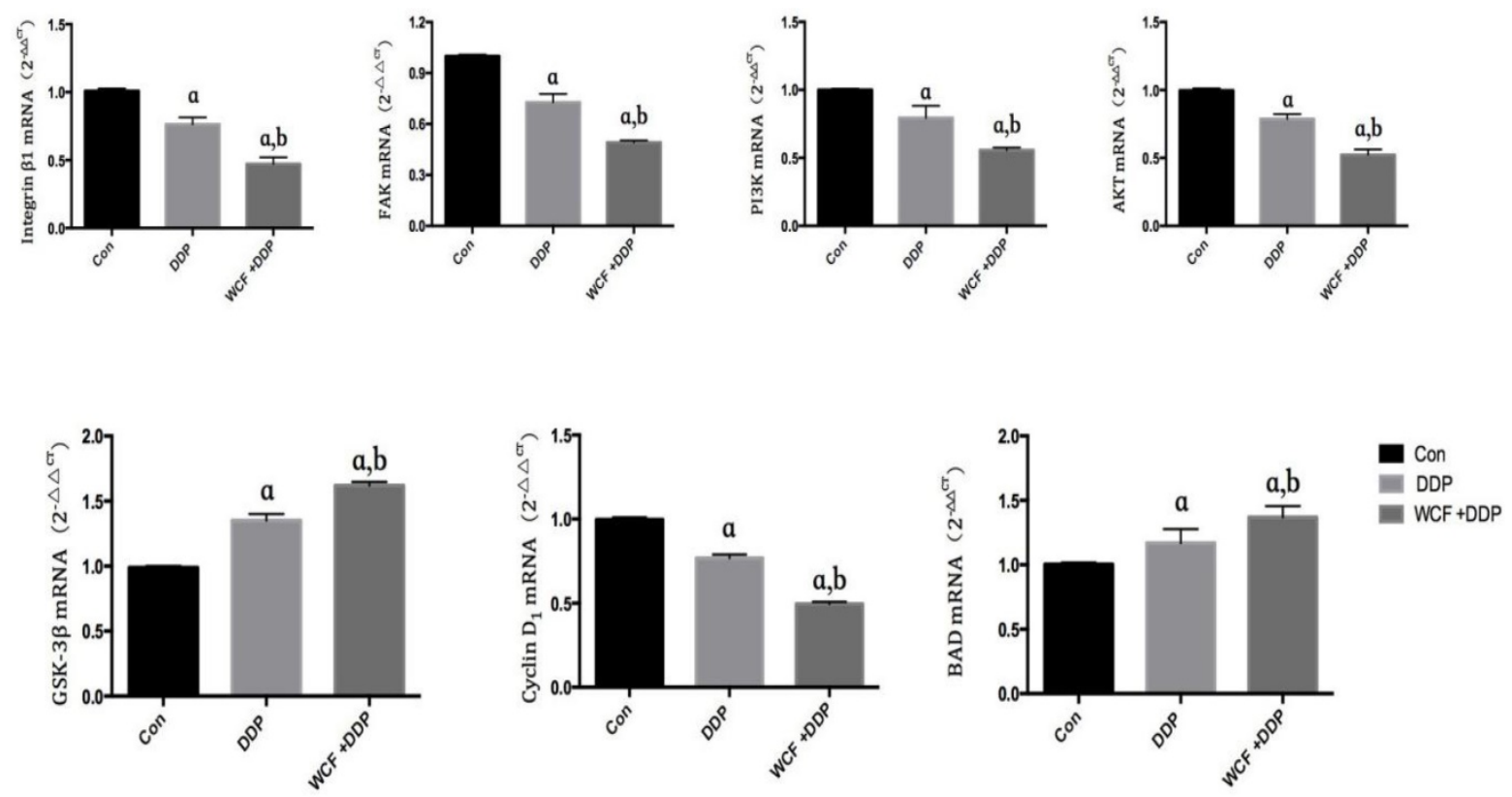

Figure 9. qRT-PCR analysis for expressions of Integrin $\beta 1$, FAK, PI3K, AKT, GSK-3ß, Cyclin DI, BAD in nude mice model. The graph represents relative densitometric intensity of each band normalized to $\beta$-actin. Values are expressed as Mean \pm SD of eight mice. aP $<0.05$, compared with control group; bP $<0.05$, compared with the DDP group.

Panax ginseng and Angelicasinensis. The combination of the four herbs utilizes the methods of warming, tonifying, purgation and elimination, and achieves the effect of dispelling cold, restoring yangqi, purgation the stagnation and clearing blood stasis.

In order to test the role of WCF, in vitro, A549 cells on three-dimensional cell culture system were incubated with DDP alone or in combination with WCF medicated serum. A549 Cells grown in Agarose formed multicellular colonies and good viability.
Agarose formed block mass and could be used as culture matrix to mimic the cell growth conditions in vivo and the three dimensional culture for cells in vitro. The cell resistance to cisplatin increased when cells formed multicelluar colonies following the culture time and reduced the sensibility of cells to DDP. The adhesion assay indicated that there were significant differences of cells in three dimensional culture to ECM proteins compared to two dimensional culture. Our results showed that A549 cells 
showed a significant reduction in cell viability after incubation with WCF. In vivo, we established a nude mouse model of the A549 cell line with Integrin $\beta 1$ overexpression, we noticed a significant reduction of the tumor sizes in the DDP treatment group and the combination of WCF and DDP treatment group as compared to that of the control groups. Taken together, results revealed that combined treatment with WCF increased the DDP cytotoxicity of A549 cells.

Then, we determined the mechanism underlying the effect of WCF on regulating cell proliferation, and flow cytometric analysis revealed that WCF generated a decrease in the percentage of cells in the G1 phase. These findings revealed that the arrest in G1 phase might be the major reason for its inhibition of proliferation in A549 cells. Moreover, we detected the expression levels of P21 and CyclinD1. P21 is a negative regulatory factor of cell cycle. The combination of P21 with CyclinD1, CyclinE and CDK2 makes cell proliferation stagnant at G1 stage, thus blocking cell cycle and inhibiting cell proliferation. Our results showed that combination of WCF and DDP treatment could reduce the expressions of P21 and CyclinD1 in A459 cells. Simultaneously, we detected the levels of apoptosis in A549 cells and found that WCF increased cell apoptosis in the cell adhesion model. Our data demonstrated that compared with those of the DDP and control groups, the cells of the combination of WCF and DDP treatment group showed a higher rate of apoptosis.

Integrin $\beta 1$ has been reported to be increased in breast cancer, ovarian cancer, non-small lung cancer, laryngeal cancer, liver cancer, and melanoma, and its expression positively correlates with viability, proliferation, and motility [19, 20, 21, 22, 23]. Recently, increasing evidence has indicated that Integrin $\beta 1$ is one of the most significant adhesion molecule that has been suggested to be involved in CAM-DR [24]. Therefore, Integrin $\beta 1$ has been suggested as a target for preventive and therapeutic approach against CAM-DR $[25,26]$. In this study, we demonstrated that DDP decreased the expression of Integrin $\beta 1$, while combination of WCF with DDP further reduced the expression of Integrin $\beta 1$ in vitro. These data indicated that depletion of Integrin $\beta 1$ enhanced the cytotoxicity of DDP.

Following the activation of Integrin $\beta 1$, focal adhesion kinase (FAK), as one of the main integrinassociated signaling and adaptor molecules, will be phosphorylated. FAK can further cooperatively interact with receptor tyrosine kinase signaling to regulate adhesion, migration, survival, proliferation, polarization, and differentiation [27]. FAK could also modulate cell apoptosis by involving PI3K (phosphatidylinositol-3-kinase) pathways [28]. The downregulation of FAK could significantly reduce Akt survival signaling, subsequently leading to apoptosis [29]. In this study, we demonstrated that DDP decreased the expression of FAK, while combination of WCF with DDP further reduced the expression of FAK in vitro. These data indicated that WCF could inhibit the expression of FAK Protein, which may be related to the mechanism of WCF induced apoptosis in lung cancer cells.

The PI3K/AKT pathway, as one of the most important downstream signaling pathways activated following by Integrin $\beta 1$ activation, was involved in multiple functions including apoptosis regulation [27]. PI3K is activated to phosphorylate phosphatidylinositol-3,4-bisphosphate (PIP2), converting it to phosphatidylinsitol-3,4,5triphosphate (PIP3). PIP3 binds to AKT and translocates to the plasma membrane, where AKT is activated by sequential phosphorylation at the T308 and S473 residues. Once activated, AKT phosphorylates many substrates to exert their functions. AKT-mediated phosphorylation negatively regulates numerous pro-apoptotic factors, including Bad [28]. The downstream of Bad may be involved in conferring Integrin $\beta 1$-mediated drug resistance [29].

The PI3K inhibitors have been used in clinical trials, but the effect of on the survival rate of cancer patients is not ideal, and the PI3K inhibitors can even promote the metastasis of tumor cells. In recent years, some traditional Chinese medicines were found to have the function of enhancing the efficacy of chemotherapy, reducing the toxicity of chemotherapy [30]. The functions of some TCM in reversing cancer MDR were through the regulation of the PI3K/AKT signaling pathway. In the present study, it was demonstrated that there was a continuous activation of PI3K/AKT in the A549 cell line and that overexpression of Integrin $\beta 1$ induced this activation. Of note, the combination of WCF and DDP treatment enhanced the inhibition effect of DDP in lung cancer cell growth via inactivating PI3K/AKT. The current study suggested that WCF could inhibit PI3K and AKT phosphorylation, which may constitute one of the mechanism by which WCF reverses CAM-DR.

Overall, we show that WCF can induce cell cycle arrest and promote the apoptosis by inhibiting Integrin $\beta 1 /$ PI3K/Akt pathway. Although our results encourage regarding $\mathrm{WCF}$ as therapeutic option for reversing cell adhesion-mediated drug resistance of lung cancer, the mechanism involved in the effect of WCF in human needs to be further determined in the future. 


\section{Conclusion}

Our findings demonstrate WCF can induce cell cycle arrest and promote the apoptosis of tumor cells which are resistant to cisplatin treatment, by inhibiting Integrin $\beta 1 / \mathrm{PI} 3 \mathrm{~K} / \mathrm{AKT}$ pathway. More importantly, the positive feedback regulatory loop of Integrin $\beta 1$ to AKT signaling pathway further adds the important role of Integrin $\beta 1$ in mediating the overall responses of WCF in this process. The in vitro and in vivo study provides an additional and a novel mechanism by which the WCF enhances the growth inhibition of DDP.

\section{Acknowledgments}

Supported by the National Natural Science Foundation of China (No.81774198, 81703839, 815738 71, 81273634), and Key Technological Innovation Project of Key Industries in Shandong Province (No.2016CYJS08A01-3, 2016CYJS08A01-4, 2016ZDJS0 7A12). The Natural Science Foundation of Shandong Province (No.ZR2014HQ032) and Shandong Province Administration of TCM also supported the work (NO.2015-016, 2017-009).

\section{Authors' Contribution}

XuMing Ji is fully responsible for the study designing, experiment adjustment, drafting, and finalizing the paper. YaNan Zhang wrote the manuscript. ZhiChun Wu carried out statistical analysis. HuaYun Yu, HuaXin Wang helped perform the analysis with constructive discussions. GuoWei Liu participated in coordination the paper. ShiJun Wang provided important suggestions on the process of the experiment, along with critical reading of the paper.

\section{Competing Interests}

The authors have declared that no competing interest exists.

\section{References}

[1] Chen W, Zheng R, Zhang S, et al. Cancer incidence and mortality in China, 2013. Cancer Letters. 2017; 401: 63-71.

[2] Chen W, Zheng R, Baade PD, Zhang S et al. Cancer statistics in China, 2015. CA Cancer J Clin. 2016; 66(2): 115-132.

[3] Mangal S, Gao W, Li T, et al. Pulmonary delivery of nanoparticle chemotherapy for the treatment of lung cancers: challenges and opportunities. Acta Pharmacol Sin. 2017; 38(6): 782-797.

[4] Lucien F, Pelletier PP, Lavoie RR, et al. Hypoxia-induced mobilization of NHE6 to the plasma membrane triggers endosome hyperacidification and chemoresistance. Nat Commun. $2017 ; 8: 15884$.

[5] Ji X M, Wu Z C, Liu G W, et al. Wenxia Changfu Formula induces apoptosis of lung adenocarcinoma in a transplanted tumor model of drug-resistance nude mice. Chin J Integr Med. 2015, 22(10): 752-758.

[6] Zhu X, Ouyang Y, Zhong F, et al. Silencing of CKIP-1 promotes tumor proliferation and cell adhesion-mediated drug resistance via regulating AKT activity in non-Hodgkin's lymphoma. Oncol Rep. 2017; 37(1): 622-630.

[7] Meads MB1, Gatenby RA, Dalton WS. Environment-mediated drug resistance: a major contributor to minimal residual disease. Nat Rev Cancer. 2009; 9(9): 665-674.
[8] Nair MG, Desai K, Prabhu JS, et al. $\beta 3$ integrin promotes chemoresistance to epirubicin in MDA-MB-231 through repression of the pro-apoptotic protein, BAD. Exp Cell Res. 2016; 346(1): 137-145.

[9] Xu X, Liu J, Shen C, et al. The role of ubiquitin-specific protease 14 (USP14) in cell adhesion-mediated drug resistance (CAM-DR) of multiple myeloma cells. Eur J Haematol. 2017; 98(1): 4-12.

[10] Jinshun Pan, Qinyi Yang, Jiaofang Shao, et al. Cyclooxygenase-2 induced $\beta 1$-integrin expression in NSCLC and promoted cell invasion via the EP1/MAPK/E2F-1/FoxC2 signal pathway. Sci Rep. 2016; 6(1): 33823.

[11] Deng QF, Su BO, Zhao YM, et al. Integrin $\beta 1$-mediated acquired gefitinib resistance in non-small cell lung cancer cells occurs via the phosphoinositide 3-kinase-dependent pathway. Oncol Lett. 2016; 11(1): 535-542.

[12] Yang X, Wu XZ. Main Anti-tumor Angiogenesis Agents Isolated From Chinese Herbal Medicines. Mini Rev Med Chem. 2015; 15(12): 1011-1023.

[13] Sui H, Fan Z Z, Li Q. Signal transduction pathways and transcriptional mechanisms of $\mathrm{ABCB} 1 / \mathrm{Pgp}$-mediated multiple drug resistance in human cancer cells. J Int Med Res. 2012; 40(2): 426-435.

[14] Guerrero-Zotano A, Mayer IA, Arteaga CL. PI3K/AKT/mTOR: role in breast cancer progression, drug resistance, and treatment. Cancer Metastasis Rev. 2016; 35(4): 1-10.

[15] Yang Z, Fang S, Di Y, et al. Modulation of NF-kB/miR-21/PTEN pathway sensitizes non-small cell lung cancer to cisplatin. Plos One. 2015; 10(3): e0121547.

[16] Lwin T, Zhao X, Cheng F, et al. A microenvironment-mediated c-Myc/miR-548m/HDAC6 amplification loop in non-Hodgkin B cell lymphomas. J Clin Invest. 2013; 123(11): 4612-4623.

[17] Tsubaki M, Takeda T, Ogawa N, et al. Overexpression of survivin via activation of ERK1/2, Akt, and NF-kappaB plays a central role in vincristine resistance in multiple myeloma cells. Leukemia research. 2015; 39(4): 445-452.

[18] Xie CQ, Zhou P, Zuo J, et al. Triptolide exerts pro-apoptotic and cell cycle arrest activity on drug-resistant human lung cancer A549/Taxol cells via modulation of MAPK and PI3K/Akt signaling pathways. Oncol Lett. 2016; 12(5): 3586-3590.

[19] Hou S, Isaji T, Hang $Q$, et al. Distinct effects of $\beta 1$ integrin on cell proliferation and cellular signaling in MDA-MB-231 breast cancer cells. Sci Rep. 2016; 6(1): 18430.

[20] Scalici JM, Harrer C, Allen A, et al. Inhibition of a4 $\beta 1$ integrin increases ovarian cancer response to carboplatin. Gynecol Oncol. 2014; 132(2): 455-461.

[21] Navab R, Strumpf D, To C, et al. Integrin a11 11 regulates cancer stromal stiffness and promotes tumorigenicity and metastasis in non-small cell lung cancer. Oncogene. 2016; 35(15): 1899-1908.

[22] Marko Klobučar, Mirela Sedić, Gehrig P , et al. Basement membrane protein ladinin- 1 and the MIF-CD44- $\beta 1$ integrin signaling axis are implicated in laryngeal cancer metastasis. BBA-Molecular Basis of Disease, 2016, 1862(10): 1938-1954.

[23] Wong KF, Liu AM, Hong W, et al. Integrin a2 $\beta 1$ inhibits MST1 kinase phosphorylation and activates Yes-associated protein oncogenic signaling in hepatocellular carcinoma. Oncotarget. 2016; 7(47): 77683-77695.

[24] Xu Z, Zou L, Ma G, et al. Integrin $\beta 1$ is a critical effector in promoting metastasis and chemo-resistance of esophageal squamous cell carcinoma. Am J Cancer Res. 2017; 7(3): 531-542.

[25] Zhan P, Liu L, Liu B, et al. Expression of integrin $\beta 1$ and its significance in squamous cell carcinoma of the cervix. Mol Med Rep. 2014, 9(6): 2473-2478.

[26] Barkan D, Chambers AF. $\beta 1$-integrin: a potential therapeutic target in the battle against cancer recurrence. Clin Cancer Res. 2011, 17(23): 7219-7223.

[27] Liang W, Ren K, Liu F, et al. Periodic mechanical stress stimulates the FAK mitogenic signal in rat chondrocytes through ERK1/2 activity. Cell Physiol Biochem. 2013; 32(4): 915-930.

[28] Sonoda Y, Matsumoto Y, Funakoshi M, Yamamoto D, Hanks SK, Kasahara T. Anti-apoptotic role of focal adhesion kinase (FAK). J Biol Chem. 2000; 275(21): 16309-16315.

[29] Liao CH, Sang S, Ho CT, Lin JK. Garcinol modulates tyrosine phosphorylation of FAK and subsequently induces apoptosis through down-regulation of Src, ERK, and Akt survival signaling in human colon cancer cells. J Cell Biochem. 2005; 96(1): 155-169.

[30] Zhao ZQ, Yu ZY, Li J, et al. Gefitinib induces lung cancer cell autophagy and apoptosis via blockade of the PI3K/AKT/mTOR pathway. Oncol Lett. 2016; 12(1): 63-68.

[31] Yip PY. Phosphatidylinositol 3-kinase-AKT-mammalian target of rapamycin (PI3K-Akt-mTOR) signaling pathway in non-small cell lung cancer. Transl Lung Cancer Res. 2015; 4(2): 165-176.

[32] Warfel N A, Kraft A S. PIM kinase (and Akt) biology and signaling in tumors. Pharmacology \& Therapeutics. 2015; 151: 41-49. 
[33] Shi Z, Song T, Wan Y, et al. A systematic review and meta-analysis of traditional insect Chinese medicines combined chemotherapy for non-surgical hepatocellular carcinoma therapy. Scientific Reports, 2017; 7(1): 4355 . 